\title{
Evaluación externa del desempeño en el tamizaje de bancos de sangre en Argentina: resultados y estrategias para mejorarlo
}

\author{
Sebastián Oknaian, ${ }^{1}$ Mirta Remesar, ${ }^{1}$ Laura Ferraro ${ }^{1}$ y Ana Emilia del Pozo ${ }^{1}$
}

RESUMEN Objetivos. Debido a la falta de un programa para la evaluación externa de la calidad del tamizaje de infecciones transmisibles por transfusión (ITT) en los bancos de sangre, el Servicio de Hemoterapia del Hospital Garrahan, de Buenos Aires, Argentina, inició en 1999 un programa continuo con el apoyo de la Organización Panamericana de la Salud (OPS) y la Asociación Argentina de Hemoterapia e Inmunohematología (AAHI).

Métodos. Se distribuyó un panel de 12 muestras con reactividad para todos los marcadores tamizados en los bancos de sangre argentinos. El panel se entregó a 52, 102 y 118 laboratorios en 1999, 2000 y 2001, respectivamente. Los laboratorios participantes se clasificaron según su desempeño en: A: menos de 2 resultados positivos falsos (RPF); B: de 2 a 3 PF; C: más de 3 $R P F ; y$ D: algún resultado negativo falso. Se efectuaron talleres con los participantes para analizar conjuntamente los resultados.

Resultados. Los porcentajes de respuesta de cada año fueron 92,3, 92,2 y 83,9, respectivamente; estos porcentajes de respuesta indican interés en las actividades de evaluación. Sin embargo, el promedio de los laboratorios con clasificación D resultó muy alto (30\%), lo que evidencia la presencia de problemas en el desempeño del tamizaje de las ITT. Esto podría asociarse con la dificultad de tomar medidas correctoras en todo el sistema, dado el elevado número de laboratorios involucrados.

Conclusiones. Se deben incluir evaluaciones de este tipo en el Programa Nacional de Sangre para el diagnóstico continuo de la situación, la toma de decisiones y el seguimiento de la calidad del tamizaje.

Palabras clave Evaluación externa de la calidad, bancos de sangre, inmunohematología, Argentina, transfusión sanguínea.

La calidad y seguridad de las transfusiones de sangre son una preocupación constante de los médicos especialistas, de las autoridades de salud y de

\footnotetext{
Hospital de Pediatría “Profesor Dr. J. P. Garrahan", Servicio de Hemoterapia, Buenos Aires, Argentina. Enviar la correspondencia a: Dr. Sebastián Oknaian, Hospital de Pediatría "Profesor Dr. J. P. Garrahan", Servicio de Hemoterapia, Combate de los Pozos 1881, CP 1245, Buenos Aires, Argentina. Fax: (54-11) 4308 5325. Correo electrónico: soknaian@garrahan.gov.ar
}

los pacientes. La seguridad de la sangre para transfusión es clave para cualquier sistema de salud moderno y la Organización Mundial de la Salud (OMS) la considera uno de los 10 problemas principales de salud a escala mundial.

En los países en desarrollo vive $80 \%$ de la población mundial, y es en ellos donde hay más problemas con la calidad de las transfusiones debido a la crisis económica que enfrentan, a las deficientes políticas económicas que no cubren todas las necesidades, al alto porcentaje de analfabetismo, y a la carencia de programas educativos en materia de salud - muchas veces se adoptan pero de manera inadecuada o restringida-, entre otras causas.

Para mejorar la calidad y seguridad de las transfusiones es importante establecer un programa de control de calidad para los bancos de sangre, cuyos elementos clave sean la gestión orga- 
nizativa, la aplicación de los estándares de calidad, la documentación adecuada y la capacitación continua. La evaluación externa de la calidad también debe formar parte de un programa de este tipo. Como parte de las evaluaciones deben efectuarse auditorías internas y externas, y es imperativo hacer la evaluación externa del desempeño del tamizaje de infecciones transmisibles por transfusión (ITT) y de la inmunohematología.

La implementación de una política de evaluación externa de la calidad en países en desarrollo exige el compromiso del gobierno, de las asociaciones de profesionales dedicados a las especialidades involucradas, y del personal que trabaja en los laboratorios (1). Los resultados de estas evaluaciones deben tenerse en cuenta al diseñar las políticas para mejorar la salud pública, pues mediante el análisis general del desempeño de los laboratorios se pueden identificar problemas e iniciar acciones correctoras o de mejoramiento que pueden reducir el riesgo residual de ITT.

Estos programas permiten también a los distintos centros participantes monitorear sus propios procedimientos y analizar los métodos empleados y sus resultados. Los errores sistemáticos cometidos por un centro solo podrán detectarse mediante su participación continua en un programa de evaluación externa.

La OMS recomienda - entre los procedimientos de evaluación externa de la calidad del tamizaje de ITT- la elaboración de controles externos con paneles de sueros $(2,3)$ y la distribución de estos a los laboratorios que realizan el tamizaje.

Los programas de evaluación externa de la calidad, tales como el del Colegio Estadounidense de Patología y el Programa de Retrovirus, de los Centros para el Control y la Prevención de Enfermedades (CDC) en Atlanta, Georgia, Estados Unidos, representan una herramienta indispensable para el control de los distintos procedimientos para la detección de marcadores de laboratorio $(4,5)$.

En 1994, el Ministerio de Salud de Brasil, junto con el Hemocentro de São Paulo, lanzó un programa para la eva- luación sistemática de la calidad del tamizaje de las ITT en los principales bancos de sangre públicos (6).

En 1995 se iniciaron en la Argentina actividades de capacitación en torno a las políticas y programas de control de la calidad, organizadas por la Asociación Argentina de Hemoterapia e Inmunohematología (AAHI) y por la Organización Panamericana de la Salud (OPS), con el objetivo de estimular la implementación de un programa de evaluación externa del tamizaje de ITT específico para bancos de sangre. Este proceso culminó en 1996 con la realización de una experiencia piloto que consistió en la preparación de 15 paneles y su distribución entre bancos de sangre de varias ciudades del interior del país para su posterior evaluación. En 1997 se estableció en la Argentina el Programa de Educación a Distancia en Sangre y Componentes Seguros, de la OMS, y a raíz de esa capacitación se hizo evidente la imperiosa necesidad de llevarlo adelante.

A pesar de intentos aislados por preparar controles externos de la calidad para el tamizaje de ITT en los bancos de sangre argentinos, no se logró implementarlos de forma sistemática $\mathrm{y}$ continua para todos los marcadores. Por esta razón, en 1999 se inició una prueba piloto en el Servicio de Hemoterapia del Hospital Garrahan, en el marco del programa de educación a distancia. Esta prueba piloto dio origen al programa que se lleva a cabo de manera continua con el apoyo de la OPS y la AAHI.

En el presente trabajo se presentan los resultados de los tres años de experiencia del Programa de Evaluación del Desempeño en el Tamizaje de ITT y se discuten las perspectivas que estos resultados permiten avizorar.

\section{MATERIALES Y MÉTODOS}

\section{Confección del panel de sueros}

El panel estuvo integrado por 12 viales con sueros reactivos a todas las pruebas de tamizaje realizadas en los bancos de sangre argentinos: anticuerpos contra el virus de la inmunodefi- ciencia humana (anti-VIH), antígeno p24 del VIH (Ag p24), anticuerpos contra el virus de la hepatitis C (antiVHC), antígeno de superficie de la hepatitis B (HBsAg), anticuerpos contra el virus linfotrópico de células $\mathrm{T}$ humanas (anti-HTLV), anticuerpos contra el antígeno nuclear del VHB (anti$\mathrm{HBc}$ ) y los agentes causales de la enfermedad de Chagas, la sífilis y la brucelosis.

Cada muestra de suero se obtuvo de una unidad de plasma reactiva, sometida a recalcificación y diálisis. Las muestras que contenían Ag p24 se obtuvieron mediante dilución del sobrenadante del cultivo del VIH (de 1:500 a 1:1000) en suero normal. Los plasmas se recalcificaron con $\mathrm{Cl}_{2} \mathrm{Ca}$ hasta una concentración final de $10 \mathrm{mM}$, y se incubaron a $37{ }^{\circ} \mathrm{C}$ hasta su coagulación. Luego se centrifugaron los sueros 15 minutos a $4{ }^{\circ} \mathrm{C}$ y se dializaron contra solución fisiológica toda la noche para eliminar el exceso de calcio. Los sueros obtenidos fueron filtrados por membranas esterilizantes y se envasaron en alícuotas en tubos estériles con tapa de rosca. Se rotularon con el código alfanumérico del lote del panel correspondiente y se almacenaron a $-80{ }^{\circ} \mathrm{C}$ hasta su distribución.

\section{Caracterización de los sueros reacti-} vos. Cada suero seleccionado se evaluó con un mínimo de dos pruebas de tamizaje adicionales y una prueba suplementaria o confirmatoria (por ejemplo, inmunoblot de Western para detectar anticuerpos anti-VIH y antiHTLV I). En el informe final de cada panel se especifican los reactivos utilizados para esta caracterización.

Estabilidad de los sueros obtenidos. Formó parte de la preparación de cada panel la realización de estudios de estabilidad de los sueros ante variaciones térmicas. El procedimiento consistió en incubar los viales de suero durante 4 días a $37{ }^{\circ} \mathrm{C}$, durante 20 días a temperatura ambiente y durante 30 días a $4{ }^{\circ} \mathrm{C}$. Los sueros también fueron sometidos a tres ciclos de congelación y descongelación, y se verificó su reactivi- 
dad por lo menos tres veces en cada período. Esto se hace con el fin de garantizar la reactividad de estos sueros aun después de su traslado a largas distancias y sin control de la temperatura.

\section{Bancos de sangre participantes}

El panel de sueros fue distribuido entre 52, 102 y 118 laboratorios en 1999,2000 y 2001, respectivamente. En el cuadro 1 se pueden observar las características de los bancos de sangre que participaron.

Una vez notificados los resultados - a los 30 días de recibido el panel-, los laboratorios participantes recibieron la clave de la reactividad de cada suero con el fin de que realizaran su propia evaluación. Con los datos aportados por todos los laboratorios participantes se elaboró un informe general que también fue remitido a cada uno.

\section{Análisis de los resultados}

Los resultados discordantes fueron utilizados para clasificar los laboratorios participantes según los siguientes criterios de desempeño:

Laboratorio A: menos de 2 resultados positivos falsos

Laboratorio B: de 2 a 3 resultados positivos falsos

Laboratorios C: más de 3 resultados positivos falsos

Laboratorios D: algún resultado negativo falso

\section{Programa para comunicar los resultados en talleres}

Se realizó un taller nacional para comunicar los resultados obtenidos y discutir con los participantes las medidas correctoras. En este taller tomaron parte el personal a cargo de la Coordinación del Programa, así como representantes de los centros participantes y de la OPS. Además, los resultados fueron notificados a las autoridades de salud junto con las medidas correctoras propuestas.

CUADRO 1. Bancos de sangre que participaron en el programa de evaluación de 1999 a 2001

\begin{tabular}{lccc}
\hline & 1999 (Panel 001) & 2000 (Panel 002) & 2001 (Panel 003) \\
\hline $\begin{array}{l}\text { Hospitales dependientes del Gobierno } \\
\text { de la Ciudad de Buenos Aires }\end{array}$ & 19 & 19 & 19 \\
$\begin{array}{l}\text { Centros privados de la Ciudad } \\
\quad \text { de Buenos Aires }\end{array}$ & 9 & 15 & 18 \\
$\begin{array}{l}\text { Otros servicios de la Ciudad } \\
\quad \text { de Buenos Aires }\end{array}$ & 2 & 2 & 5 \\
$\begin{array}{l}\text { Bancos de sangre del resto del país } \\
\quad \text { Total }\end{array}$ & 22 & 66 & 76 \\
\hline
\end{tabular}

CUADRO 2. Desempeño de todos los laboratorios que participaron en el programa de evaluación de 1999 a 2001

\begin{tabular}{|c|c|c|c|c|c|c|}
\hline & \multicolumn{2}{|c|}{1999 (Panel 001) } & \multicolumn{2}{|c|}{2000 (Panel 002) } & \multicolumn{2}{|c|}{2001 (Panel 003) } \\
\hline & No. & $\%$ & No. & $\%$ & No. & $\%$ \\
\hline Laboratorios A & 31 & 64,6 & 50 & 53,2 & 41 & 41,4 \\
\hline Laboratorios B & 1 & 2,1 & 10 & 10,6 & 14 & 14,2 \\
\hline Laboratorios C & 5 & 10,4 & 6 & 6,4 & 13 & 13,1 \\
\hline Laboratorios D & 11 & 22,9 & 28 & 29,8 & 31 & 31,3 \\
\hline Total & 48 & & 94 & & 99 & \\
\hline
\end{tabular}

\section{RESULTADOS}

El número de laboratorios participantes aumentó de 52 en 1999 a 102 y 118 en los años 2000 y 2001, respectivamente (cuadro 1). Los porcentajes de respuesta, es decir, la proporción de los laboratorios que enviaron sus resultados fue de $92,3 \%$ en $1999,92,2 \%$ en 2000 y 83,9\% en 2001 .

No se advirtieron diferencias en el desempeño global de los laboratorios (cuadro 2). Sin embargo, si se agrupan los resultados de la evaluación de los laboratorios que participaron durante los tres años en que se desarrolló el programa, se observa una disminución en el porcentaje de laboratorios $\mathrm{D}$ en el último año. Los resultados de los laboratorios que participaron en los paneles de todos los tres años se muestran en el cuadro 3.

A los talleres asistieron 40 bioquímicos especialistas de los laboratorios participantes. En esos talleres se analizaron los resultados; se revisaron los tipos de errores (de transcripción, interpretación u omisión, deficiencias en el mantenimiento de los equipos, etc.); se ofreció capacitación para la elaboración de controles internos y la elaboración de la documentación necesaria en los laboratorios (tales como registros, manuales de procedimientos operativos estándares); y se realizaron encuestas de evaluación dirigidas a conocer la situación de los laboratorios, la opinión sobre los talleres y sus propuestas.

Las encuestas demostraron la falta de procedimientos de control de calidad, como podrían ser la utilización de controles internos y el mantenimiento preventivo de los equipos.

Una de las conclusiones más importantes del taller fue el reconocimiento de que la mayoría de las discrepancias se debieron al desempeño deficiente de los laboratorios, más que a la deficiencia de los reactivos utilizados en el tamizaje de las ITT.

\section{DISCUSIÓN}

Los talleres facilitaron el análisis conjunto de los resultados, su discu- 
CUADRO 3. Desempeño de solamente los laboratorios que participaron en todos los paneles distribuidos de 1999 a 2001

\begin{tabular}{|c|c|c|c|c|c|c|}
\hline & \multicolumn{2}{|c|}{1999 (Panel 001) } & \multicolumn{2}{|c|}{2000 (Panel 002) } & \multicolumn{2}{|c|}{2001 (Panel 003) } \\
\hline & No. & $\%$ & No. & $\%$ & No. & $\%$ \\
\hline Laboratorios A & 25 & 61,0 & 25 & 61,0 & 21 & 51,2 \\
\hline Laboratorios B & 1 & 2,4 & 4 & 9,8 & 7 & 17,1 \\
\hline Laboratorios C & 4 & 9,8 & 1 & 2,4 & 6 & 14,6 \\
\hline Laboratorios D & 11 & 26,8 & 11 & 26,8 & 7 & 17,1 \\
\hline Total & 41 & & 41 & & 41 & \\
\hline
\end{tabular}

sión y la ampliación de los conocimientos relacionados con los procedimientos de control de calidad que deben adoptarse en cada banco de sangre. El Programa fomentó la comunicación entre los servicios para la realización de consultas y la colaboración en futuros proyectos de tamizaje de las ITT.

Los porcentajes de respuesta indican el gran interés de los bancos de sangre en las actividades de evaluación del desempeño. Un elemento fundamental —además de la utilización de los controles externos para evaluar el desempeño o la competencia técnica $(7,8)$ - , es el monitoreo de las pruebas de tamizaje con controles internos (controles que no se incluyen en los sistemas comerciales) (9-11), por lo que la capacitación en su preparación es esencial.

El análisis de los resultados de desempeño refleja que el número de laboratorios con clasificación $\mathrm{D}$ durante los tres años es muy alto, de aproximadamente $30 \%$, lo que evidencia la existencia de graves problemas en el tamizaje de las ITT. Como consecuencia, existe un alto riesgo de transmisión de infecciones a los pacientes transfundidos.

Entre los bancos de sangre que participaron durante los tres años ininterrumpidamente, el porcentaje de laboratorios $\mathrm{D}$ disminuyó el último año, a pesar de que el número de laboratorios cuyo desempeño puede considerarse bueno (A y B) no aumentó. Estos resultados indican que sigue siendo necesario capacitar a todos los laboratorios del sistema para introducir programas de control de la calidad de una manera eficaz y poner en práctica las medidas correctoras específicas que sean pertinentes, de acuerdo con los hallazgos o la solicitud de los participantes. Sin embargo, esto es algo difícil, dada la gran dispersión de los servicios.

El programa de evaluación debe vincularse al monitoreo de la elaboración y a la revisión constante de los manuales de procedimientos, que son parte de los documentos del programa de calidad de los servicios. Los manuales operativos estándares permiten establecer una forma de trabajo uniforme. Por su parte, la evaluación externa no puede estar desvinculada del desarrollo de programas de capacitación continua que contemplen los hallazgos del análisis de los resultados de la evaluación.

Los resultados obtenidos respaldan la necesidad de incluir la evaluación del desempeño en el Programa Nacional de Sangre como una herramienta indispensable y obligatoria para el diagnóstico continuo de la situación, la toma de decisiones orientadas a corregir los problemas que se detectan y el seguimiento de la calidad de los laboratorios dedicados al tamizaje de ITT.

\section{REFERENCIAS}

1. World Health Organization. Requirements and guidance for external quality assessment schemes for health laboratories. Geneva: WHO; 1999. (WHO/DIL/LAB/99.2).

2. Organización Panamericana de la Salud. Manual de procedimientos de control de calidad para los laboratorios de serología de los bancos de sangre. Washington, D.C.: OPS; 1994. (PAHO/HPC/HCT/94.21).

3. Organización Mundial de la Salud. Garantía de calidad en el diagnóstico serológico de la infección por el virus de la inmunodeficiencia humana. Manual de Laboratorio. Buenos Aires: OMS; 1995. (Publicación No 42). World Health Organization, Quality Management Project For Blood Transfusion Services, Blood Transfusion Safety, 2000.

4. Schalla WO, Hearn TL, Taylor RN, Eavenson E, Valdiserri RO, Essien JD. CDC's Model Performance Evaluation Program: assessment of the quality of laboratory performance for
HIV-1 antibody testing. Public Health Rep 1990;105:167-171.

5. Williams LO, Blumer SO, Schalla WO, Robinson $\mathrm{PH}$, Handsfield JH, Fehd RJ et al. Laboratory performance in HTLV-I/II analysis. Transfusion 2000;40:1514-1521.

6. Saez-Alquezar A, Otani MM, Sabino EC, Ribeiro-dos-Santos G, Salles N, Chamone DF. Evaluation of the performance of Brazilian blood banks in testing for Chagas' Disease. Vox Sang 1998;74:228-231.

7. International Federation of Clinical Chemistry. Fundamentals for external quality assessment (EQA). Strasbourg: IFCC; 1996.

8. ISO. Proficiency testing by interlaboratory comparisons. Part. 1. Development and operation of proficiency testing schemes. ISO Guide 43-1; 1996. (ISO/CASCO 331).

9. Council of Europe Publishing. Guide to the preparation, use and quality assurance of blood components. Quinta edición. CEP; 1999.
10. World Health Organization. Quality systems for medical laboratories. Guidelines for implementation and monitoring. Alexandria, Egypt: WHO Regional Office for the Eastern Mediterranean; 1995. (Serie 14).

11. World Health Organization. Guidelines for organizing national EQASs for HIV serological testing: UNAIDS; 1996.

Manuscrito recibido el 23 de agosto de 2002. Aceptado para publicación, tras revisión, el 24 de enero de 2003. 
ABSTRACT Objectives. Because there was no program for the external evaluation of the quality of the screening for transfusion-transmitted infections (TTIs) in blood banks in Argentina, in 1999 the Hemotherapy Service of Garrahan Hospital, in Buenos Aires, launched an ongoing external evaluation program, with the support of the Pan American Health Organization and the Argentine Hemotherapy and Immunohematology Association.

Methods. A panel of 12 samples that were reactive to all the markers screened for in Argentine blood banks was distributed. The panel was delivered to 52 laboratories in 1999, 102 laboratories in 2000, and 118 laboratories in 2001. The participating laboratories were classified into one of four categories according to their performance: A: 0 or 1 false positive (FP) results; B: 2 or 3 FPs; C: 4 or more FPs; and D: at least 1 false negative result. Workshops were held with the participants in order to jointly analyze the results.

Results. Out of the laboratories that received the panel of samples, the percentage of laboratories that sent in their results was $92.3 \%$ in $1999,92.2 \%$ in 2000 , and $83.9 \%$ in 2001. These response levels demonstrate the interest in evaluation activities. However, the annual average percentage of the laboratories that received a " $\mathrm{D}$ " classification was very high $(30 \%)$, which indicates that there are problems in the performance of TTI screening. This poor showing could be related to the difficulty in taking corrective measures throughout the system, given the large number of laboratories involved.

Conclusions. Evaluations of this type should be included in Argentina's National Blood Program as a tool for the ongoing assessment of the blood banking situation, for decision-making, and for monitoring the quality of screening.

\title{
Premio Abraham Horwitz al liderazgo en la salud interamericana
}

Este premio, que fue establecido en 1975 por la Fundación Panamericana de la Salud y Educación, es un reconocimiento a aquellas personas cuyos logros en el campo de la salud pública han redundado en mejoras importantes en la vida y salud de las poblaciones americanas. Los candidatos pueden ser individuos - activos o jubilados - o grupos que han destacado por su labor científica o pedagógica en cualquier rama de la salud interamericana. La propuesta debe acompañarse de una carta de presentación; el formulario de propuesta del Comité del Premio Horwitz; la información personal del candidato y de la persona que lo postula; una descripción detallada de los logros específicos que ameritan la concesión del premio, destacando su importancia en la Región y un currículum vitae actualizado. El galardonado recibirá un diploma de honor y US\$ 5000 en efectivo. de 2003

La fecha límite para la recepción de las candidaturas es el 15 de mayo

\author{
Información: \\ Comité Premio Abraham Horwitz \\ Fundación Panamericana de la Salud y Educación \\ 525 23rd Street, N.W. \\ Washington, DC 20037 \\ Teléfono: (202) 974-3416 • Fax: (202) 974-3636 \\ Correo electrónico: pahef@paho.org
}

